\title{
Adaptive Backstepping Control and Tracking Control for New Chaotic Dynamical System
}

\author{
Qian Zhang and Junyi Zhao
}

\begin{abstract}
Chaos is a very interesting nonlinear phenomenon, and often encountered in many dynamics. Hence, Chaos control problem has been one of the main concerns of researchers wishing to inspect the properties of such systems. This paper focuses on the adaptive control problem and tracking control problem for a new chaotic dynamical system with unknown parameter. Based on a modified backstepping technique and adaptive control method, some chaos controllers are developed for such systems. In comparison with previous methods, the present control technique can improve the speed of parameter identification. The stability analysis in the closed-loop system is given by the Lyapunov method. Finally, numerical simulations are provided to verify the feasibility and effectiveness of the designed controller.
\end{abstract}

Index Terms-Chaos control, adaptive control, backstepping, Lyapunov.

\section{INTRODUCTION}

Chaos is a very interesting nonlinear phenomenon, and often encountered in many dynamics, such as signal generator [1], secure communication [2], circuits [3], lasers [4], etc. Hence, Chaos control problem has been one of the main concerns of researchers wishing to inspect the properties of such systems [5]. Nowadays, many techniques have been proposed to control chaos, including OGY method [6], linear state space feedback [7], adaptive control [8], etc. Some methods are based on knowing the system structure and parameters. But in practical situations, there are many uncertain systems. Therefore, the derivation of an adaptive controller for the control of chaotic systems with unknown system parameters is an important issue. This issue involves the identification of the unknown parameters and the approach of controlling chaotic system. And adaptive backstepping control has been one of the most effective approaches for the design of nonlinear system.

In this work we investigate the problem of chaos control and tracking control for a new chaotic dynamical system and propose a modified backstepping technique and adaptive control method. In comparison with previous methods, the present control technique can improve the speed of parameter identification. The stability analysis in the closed-loop system is given by the Lyapunov method. Finally, numerical simulations are provided to verify the feasibility and effectiveness of the designed controller.

Manuscript received February 25, 2013; revised April 16, 2013.

Qian Zhang was with the East China University of Science and Technology, shanghai, China. He is now with the Computer Center, East China Normal University, Shanghai, China (e-mail: zq5117@126.com).

Junyi Zhao is with the Computer Center, East China Normal University, Shanghai, China (e-mail: neftwoz@yahoo.com.cn).

\section{SYSTEM DESCRIPTION}

The new chaotic dynamical system [9] is described by:

$$
\left\{\begin{array}{l}
\dot{x}=a(y-x) \\
\dot{y}=b x-x z \\
\dot{z}=x y+c z
\end{array}\right.
$$

where $a, b$ and $c$ are three unknown parameters. When the parameters $a=10, b=16, c=-1$, system (1) displays a chaotic attractor (see Fig. 1).

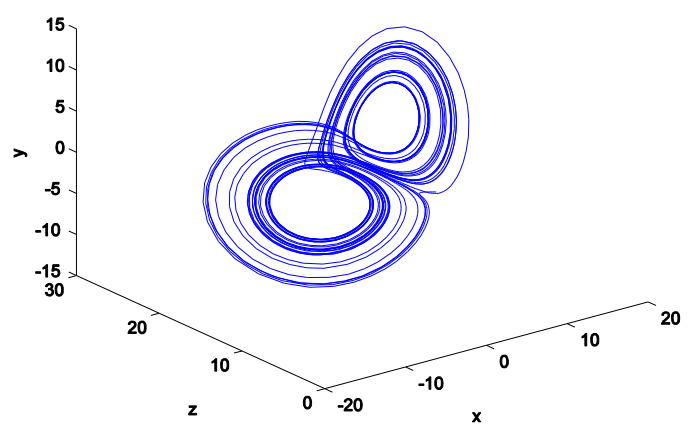

Fig. 1. The new dynamical system at $a=10, b=16, c=-1$

The system (1) belongs to generalized Lorenz system. Since the eigenvalues of the corresponding Jacobians of the new system (1) are different from the Lorenz system [10], system (1) and Lorenz system are not topological equivalent.

\section{CONTROLling CHAOTIC SySTEM AND IDENTIFICATION OF THE UNKNOWN PARAMETER}

In this section, we will use a modified backstepping approach to design a controller. We will add an extra control parameter in our controller to improve the speed of parameter identification.

Assume that all parameters $a, b, c$ in system (1) are unknown and need to be identified, we add a control input $u$ to the third equation of system (1). Now, we rewrite system (1) as the following:

$$
\left\{\begin{array}{l}
\dot{x}=a(y-x) \\
\dot{y}=b x-x z \\
\dot{z}=x y+c z+u
\end{array}\right.
$$

Then we will identify the three parameters and control the uncertain system (2). At each step, we will construct control function $\alpha_{i}$ via selecting an appropriate Lyapunov function $V_{i}$ (stabilizing function).

We define three error variables as the following: 


$$
\left\{\begin{array}{l}
\bar{x}=x \\
\bar{y}=y-\alpha_{1} \\
\bar{z}=z-\alpha_{2}
\end{array}\right.
$$

Step 1:

Calculating the time derivative of the 1 st equation $\bar{x}=x$, we have

$$
\dot{\bar{x}}=a\left(\bar{y}+\alpha_{1}-\bar{x}\right)
$$

We define a Lyapunov function as

$$
V_{1}(\bar{x})=\frac{1}{2} \bar{x}^{2}
$$

Taking the time derivative of (5), we get

$$
\dot{V_{1}}=\bar{x} \dot{\bar{x}}=-a \bar{x}^{2}+a \bar{x} \bar{y}+a \bar{x} \alpha_{1}
$$

We choose

$$
\alpha_{1}=p_{1} \bar{x}-p_{2} \bar{y}
$$

where $p_{1} \in(0,1), p_{2} \in(0,1), p_{2}$ is the extra control parameter in our controller to improve the speed of parameter identification.

We can rewrite (6) as the following:

$$
\dot{V}_{1}=-a\left(1-p_{1}\right) \bar{x}^{2}+a\left(1-p_{2}\right) \bar{x} \bar{y}
$$

\section{Step 2:}

Calculating the time derivative of the 2 nd equation $\bar{y}=y-\alpha_{1}$, we have

$$
\begin{aligned}
\dot{\bar{y}} & =\dot{y}-\dot{\alpha_{1}} \\
& =b x-x z-p_{1} \dot{\bar{x}}+p_{2} \dot{\bar{y}} \\
& =\frac{1}{1-p_{2}}\left[b \bar{x}-\bar{x}\left(\bar{z}+\alpha_{2}\right)-a p_{1}\left(\bar{y}-\bar{x}+p_{1} \bar{x}-p_{2} \bar{y}\right)\right]
\end{aligned}
$$

Assume the errors of three parameters are $\tilde{a}, \tilde{b}, \tilde{c}$ as the following:

$$
\left\{\begin{array}{l}
\tilde{a}=\hat{a}-a \\
\tilde{b}=\hat{b}-b \\
\tilde{c}=\hat{c}-c
\end{array}\right.
$$

where $\hat{a}, \hat{b}, \hat{c}$ are three identification values.

We introduce a Lyapunov function as

$$
V_{2}(\bar{x}, \bar{y})=V_{1}+\frac{1}{2} \bar{y}^{2}+\frac{1}{2} \tilde{a}^{2}+\frac{1}{2} \tilde{b}^{2}
$$

Taking the time derivative of (11), we get

$$
\begin{aligned}
& \dot{V_{2}}=\dot{V}_{1}+\bar{y} \dot{\bar{y}}+\tilde{a} \dot{\tilde{a}}+\tilde{b} \dot{\tilde{b}} \\
&=-a\left(1-p_{1}\right) \bar{x}^{2}+a\left(1-p_{2}\right) \bar{x} \bar{y}+\tilde{a} \dot{\tilde{a}}+\tilde{b} \dot{\tilde{b}} \\
&+\frac{\bar{y}}{1-p_{2}}\left[b \bar{x}-\bar{x} \alpha_{2}-a p_{1}(\bar{y}-\bar{x}+p \bar{x}-p \bar{y})\right] \\
&=-a\left(1-p_{1}\right) \bar{x}^{2}-a p_{1} \bar{y}^{2}-\frac{\bar{x} \bar{y} \bar{z}}{1-p_{2}} \\
&+\bar{x} \bar{y}\left\{\hat{a}\left(1-p_{2}\right)+\frac{1}{1-p_{2}}\left[\hat{b}+\hat{a}\left(p_{1}-p_{1}{ }^{2}\right)-\alpha_{2}\right]\right\} \\
&+\tilde{a}\left\{\dot{\tilde{a}}-\bar{x} \bar{y}\left[\left(1-p_{2}\right)+\frac{p_{1}-p_{1}{ }^{2}}{1-p_{2}}\right]\right\}+\tilde{b}\left(\dot{\tilde{b}}-\frac{\bar{x} \bar{y}}{1-p_{2}}\right)
\end{aligned}
$$

We choose

$$
\begin{gathered}
\alpha_{2}=\hat{b}+\hat{a}\left(p_{1}-p_{1}{ }^{2}\right)+\hat{a}\left(1-p_{2}\right)^{2} \\
\dot{\tilde{a}}=\bar{x} \bar{y}\left[\left(1-p_{2}\right)+\frac{p_{1}-p_{1}{ }^{2}}{1-p_{2}}\right]-m \tilde{a} \\
\dot{\tilde{b}}=\frac{\bar{x} \bar{y}}{1-p_{2}}-n \tilde{b}
\end{gathered}
$$

where $m, n$ are Control constants and $m>0, n>0$.
And we can rewrite (12) as the following:

$\dot{V_{2}}=-a\left(1-p_{1}\right) \bar{x}^{2}-a p_{1} \bar{y}^{2}-\frac{\bar{x} \bar{y} \bar{z}}{1-p_{2}}-m \tilde{a}^{2}-n \tilde{b}^{2}$

\section{Step 3:}

Calculating the time derivative of the 3rd equation $\bar{z}=z-\alpha_{2}$, we have

$$
\begin{aligned}
\dot{\bar{z}} & =\dot{z}-\dot{\alpha_{2}} \\
& =x y+c z+u-\dot{\alpha_{2}} \\
& =\bar{x}\left(\bar{y}+p_{1} \bar{x}-p_{2} \bar{y}\right)+c z+u-\frac{\partial \alpha_{2}}{\partial \hat{a}} \dot{\hat{a}}-\frac{\partial \alpha_{2}}{\partial \hat{b}} \dot{\hat{b}}
\end{aligned}
$$

We can define a Lyapunov function as

$$
V_{3}(\bar{x}, \bar{y}, \bar{z})=V_{2}+\frac{1}{2} \bar{z}^{2}+\frac{1}{2} \tilde{c}^{2}
$$

Taking the time derivative of (18), we get

$$
\begin{aligned}
\dot{V}_{3} & =\dot{V}_{2}+\bar{z} \dot{\bar{z}}+\tilde{c} \dot{\tilde{c}} \\
& =-a\left(1-p_{1}\right) \bar{x}^{2}-a p_{1} \bar{y}^{2}-\frac{\bar{x} \bar{y} \bar{z}}{1-p_{2}}-m \tilde{a}^{2}-n \tilde{b}^{2}+\tilde{c} \dot{\tilde{c}} \\
& +\bar{z}\left[\bar{x}\left(\bar{y}+p_{1} \bar{x}-p_{2} \bar{y}\right)+c z+u-\frac{\partial \alpha_{2}}{\partial \hat{a}} \dot{\hat{a}}-\frac{\partial \alpha_{2}}{\partial \hat{b}} \dot{\hat{b}}\right] \\
& =-a\left(1-p_{1}\right) \bar{x}^{2}-a p_{1} \bar{y}^{2}-m \tilde{a}^{2}-n \tilde{b}^{2} \\
& +\bar{z}\left[p_{1} \bar{x}^{2}+\hat{c} z+u+\frac{p_{2}{ }^{2}-2 p_{2}}{1-p_{2}} \bar{x} \bar{y}-\frac{\partial \alpha_{2}}{\partial \hat{a}} \dot{\hat{a}}-\frac{\partial \alpha_{2}}{\partial \hat{b}} \dot{\hat{b}}\right] \\
& +\widetilde{c}(\dot{\tilde{c}}-z \bar{z})
\end{aligned}
$$

We choose

$$
\begin{gathered}
u=\frac{\partial \alpha_{2}}{\partial \hat{a}} \dot{\hat{a}}+\frac{\partial \alpha_{2}}{\partial \hat{b}} \dot{\hat{b}}-p_{1} \bar{x}^{2}-\hat{c} z-\frac{p_{2}{ }^{2}-2 p_{2}}{1-p_{2}} \bar{x} \bar{y}-q \bar{z} \\
\dot{\tilde{c}}=z \bar{z}-k \tilde{c}
\end{gathered}
$$

where $q, k$ are control constants and $q>0, k>0$.

And we can rewrite (19) as the following:

$$
\begin{aligned}
& \dot{V_{3}}=-a\left(1-p_{1}\right) \bar{x}^{2}-a p_{1} \bar{y}^{2}-q \bar{z}^{2}-m \tilde{a}^{2}-n \tilde{b}^{2}- \\
& \quad k \tilde{c}^{2}
\end{aligned}
$$

At this point, the system (2) state can be stabilized and the unknown parameters can be identified under the action of the controller $u$ (20) and the parameters adaptive rate (14) (15) (21).

Stability Analysis: Lyapunov function $V=V_{3}$

Taking the time derivative, we get

$$
\begin{aligned}
& \dot{V}=\dot{V}_{3}=-a\left(1-p_{1}\right) \bar{x}^{2}-a p_{1} \bar{y}^{2}-q \bar{z}^{2}-m \tilde{a}^{2}- \\
& n \tilde{b}^{2}-k \tilde{c}^{2} \leq 0
\end{aligned}
$$

Barbalat Lemma [11], the system (2) is asymptotically stable.

\section{TRACKING ANY DESIRED TRAJECTORY}

In this section, we will apply the above technique to design a tracking controller for system (1). To simplify the problem in the present section, we assume that the three parameters $a, b$ and $c$ are seen as known parameters and set the output of the system $\xi=x$.

We can add a control input $u_{2}$ to the second equation of system (1). Now, we rewrite system (1) as the following:

$$
\left\{\begin{array}{l}
\dot{x}=a(y-x) \\
\dot{y}=b x-x z+u_{2} \\
\dot{z}=x y+c z
\end{array}\right.
$$

We define error variables as the following: 


$$
\left\{\begin{array}{l}
\bar{x}=x-y_{d} \\
\bar{y}=y-\alpha_{1}
\end{array}\right.
$$

where $y_{d}$ is any desired trajectory.

Step 1:

Defining a Lyapunov function as

$$
V_{1}(\bar{x})=\frac{1}{2} \bar{x}^{2}
$$

Taking the time derivative of (23), we get

$$
\begin{aligned}
& \dot{V}_{1}=\bar{x} \dot{\bar{x}} \\
& \quad=\left(x-y_{d}\right)\left(\dot{x}-\dot{y}_{d}\right) \\
& \quad=-\left(x-y_{d}\right)^{2}+\left(x-y_{d}\right)\left[x-y_{d}+a(y-x)-\dot{y}_{d}\right]
\end{aligned}
$$

To make $\dot{V_{1}}$ be negative definite, we choose

$$
\alpha_{1}=y=x+\frac{1}{a}\left(-x+y_{d}+\dot{y}_{d}\right)
$$

We can rewrite (27) as the following:

$$
\dot{V}_{1}=-\left(x-y_{d}\right)^{2}
$$

\section{Step 2:}

Defining a Lyapunov function as

$$
V_{2}(\bar{x}, \bar{y})=V_{1}+\frac{1}{2} \bar{y}^{2}
$$

Taking the time derivative of (30), we get

$$
\begin{aligned}
\dot{V}_{2}= & \dot{V}_{1}+\bar{y} \dot{\bar{y}} \\
= & -\left(x-y_{d}\right)^{2}+\left(y-x-\frac{-x+y_{d}+y_{d}}{a}\right)(\dot{y}-\dot{x}- \\
& \left.\frac{-\dot{x}+y_{d}+y_{d}^{\prime}}{a}\right) \\
= & -\left(x-y_{d}\right)^{2}-\left(y-x-\frac{-x+y_{d}+y_{d}}{a}\right)^{2} \\
& +\left(y-x-\frac{-x+y_{d}+y_{d}}{a}\right)\left[u_{2}+b x-x z+(2-a)(y-\right. \\
& x)+x-y d-2 y d-y d a
\end{aligned}
$$

To make $\dot{V_{2}}$ be negative definite, we choose

$$
u_{2}=-b x+x z+(a-2)(y-x)-\frac{x-y_{d}-2 y_{d}-\ddot{y_{d}}}{a}
$$

We can rewrite (31) as the following:

$$
\dot{V}_{2}=-\left(x-y_{d}\right)^{2}-\left(y-x-\frac{-x+y_{d}+y_{d}}{a}\right)^{2} \leq 0
$$

Obtained above controller $u_{2}$ (32), we can guarantee $\dot{V}_{2}$ negative definite, which enables the output of the system (24) follow any trajectory $y_{d}$ steadily.

\section{Simulations}

In order to verify the feasibility and effectiveness of the above method, we use a fourth-order Runge-Kutta method algorithm for the numerical simulation in this section. We assume that three parameters are chosen to $a=10 b=$ $16 c=-1$ in all simulations, so that the new system exhibits a chaotic behavior. We also assume that $q=100, m=n=$ $k=30, p_{1}=0.62$. If our extra control parameter $p_{2}=0$, the controller $u(20)$ is the same as the one that is obtained by the previous backstepping method. At this point, we choose the extra control parameter $p_{2}=0.8$. Fig. 2 illustrates the parameter identification in this paper, and the design of parameters adaptive law made a significant effect. The red lines represent $p_{2}=0.8$, while blue lines represent $p_{2}=0$ in the Fig. 2.

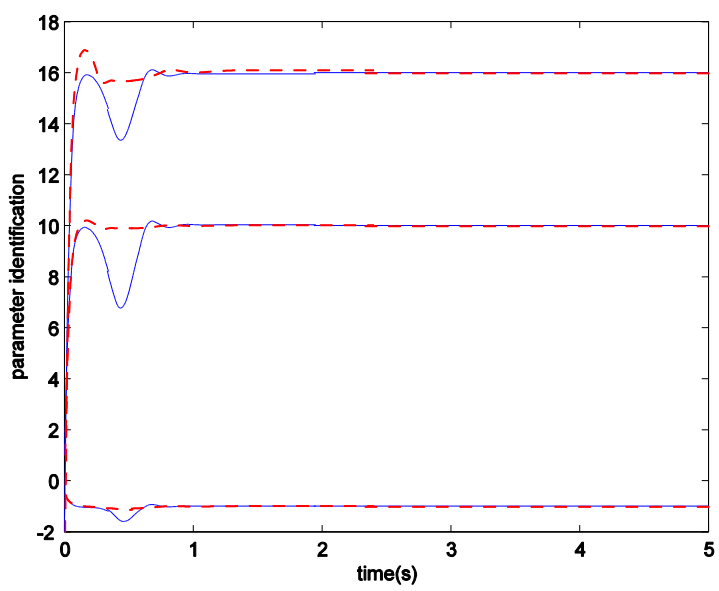

Fig. 2. Identification of the unknown parameters.

Fig. 2 demonstrates that the extra control parameter $p_{2}$ in our controller has improved the speed of parameter identification.

Fig. 3 shows the system state variables $x, y, z$, soon reached theoretically expected boundary point.

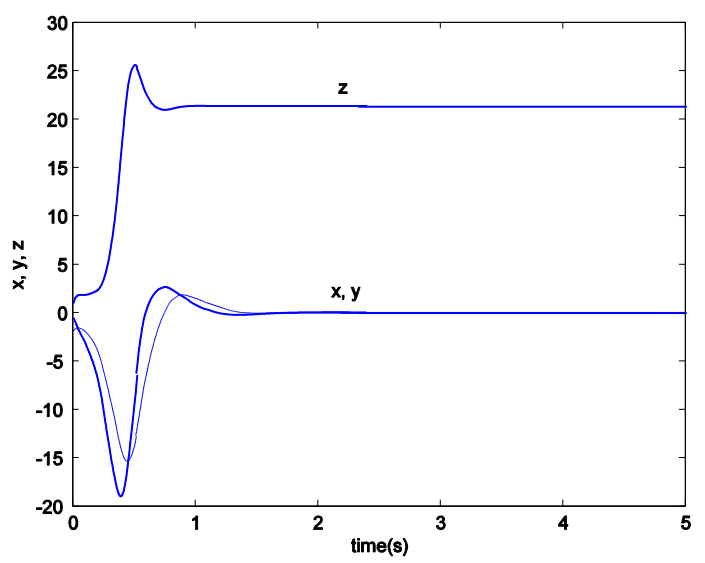

Fig. 3. The system state variables $x, y, z$

We select $y_{d}=0.5[\sin (t)+\sin (0.5 t)]$ to verify the effectiveness of the tracking controller $u_{2}$ (31).

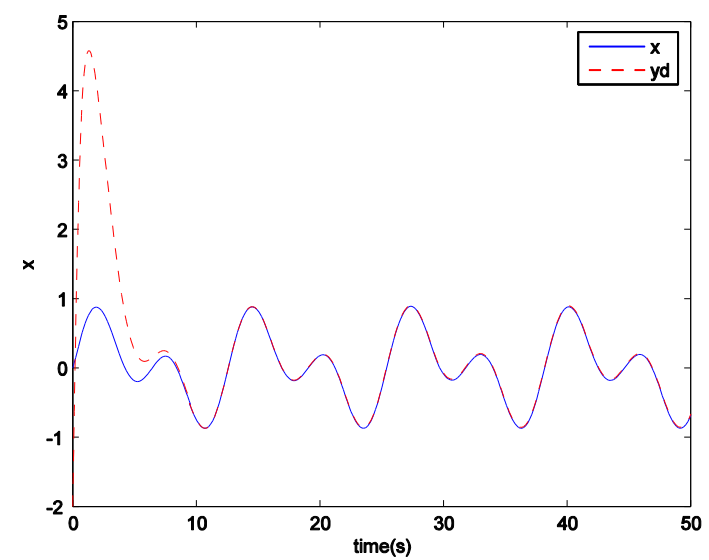

Fig. 4. Tracking $y_{d}=0.5[\sin (t)+\sin (0.5 t)] \times$ phase plane

Fig. 4 shows the output $\mathrm{x}$ of the system (24) can follow the trajectory $y_{d}$ steadily. From Figures 2,3 and 4, it can be concluded that the chaos control and tracking control for the 
new chaotic dynamical system (1) is achieved via adaptive control.

\section{CONCLUSION}

In this paper, a modified backstepping technique and adaptive control method are proposed for controlling a new chaotic dynamical system (1). Based on the Lyapunov stability theory and above method, the two controllers $u$ (20) and $u_{2}$ (32) are designed to achieve chaos control and tracking control. An appropriate extra control parameter $p_{2}$ in our controller (20) can improve the speed of parameter identification. Numerical simulations show that the proposed method work effectively.

\section{REFERENCES}

[1] M. Gonzalez and D. Mendez, "Digital noise generator based on Bernoulli chaotic map," Applied Mechanics and Materials, vol. 152, pp. 1869-1873, 2012.

[2] X. Y. Wang and M. J. Wang, "A chaotic secure communication scheme based on observer," Communications in Nonlinear Science and Numerical Simulation, vol. 14, pp. 1502-1508, 2009.
[3] X. F. Wang, "Generating chaos in Chua's circuit via time-delay feedback," Circuits and Systems, vol. 48, pp. 1151-1156, 2001.

[4] J. R. Terry and K. S. Thornburg, "Synchronization of chaos in an array of three lasers," Physical Review E, vol. 59, pp. 4036-4043, 1999.

[5] J. Lü, J. Lu, and S. Chen, Chaotic time series analysis and its application, Wuhan University Press, vol. 4, pp. 570-578, 2003.

[6] E. Ott, C. Grebogi, and J. A. Yorke, "Controlling chaos," Physical Review Letters, vol. 64, pp. 1196-1199, 1990.

[7] G. Chen and X. Dong, "On feedback control of chaotic continuous-time systems," Circuits and Systems, vol. 40, pp. 591-601, 1993.

[8] Y. J. Cao, "A nonlinear adaptive approach to controlling chaotic oscillators," Physics Letter, vol. 270, pp. 171-176, 2000.

[9] W. N. Zhou and Y. H. Xu, "On dynamics analysis of a new chaotic attractor," Physics Letters A, vol. 372, pp. 5773-5777, 2008.

[10] E. N. Lorenz, "Deterministic nonperiodic flow," J. Atmos. Sci, vol. 20 , pp. 130-141, 2003.

[11] R. Mukherjee and D. Ghen, "Asymptotic stability theorem for autonomous systems," Journal of Guidance, Control, and Dynamics, vol. 16, pp. 961-963, 1993.

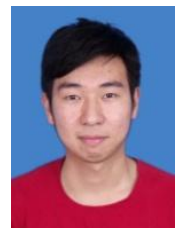

Qian Zhang was born in Shanghai, September 1989. He graduated from East China University of Science and Technology, majoring in electrical engineering and automation. Now he is studying for a master's degree in East China Normal University. His major field of study is embedded systems and control engineering. 